\title{
6D Workspace Constraints for Physical Human-Robot Interaction using Invariance Control with Chattering Reduction
}

\author{
Melanie Kimmel, Martin Lawitzky and Sandra Hirche \\ Institute of Automatic Control Engineering \\ Technische Universität München \\ 80290 Munich, Germany \\ Email: melanie.kimmel@mytum.de,ml@tum.de, hirche@tum.de
}

\begin{abstract}
For safety in physical human-robot interaction (pHRI) the robot motion must be restricted to an admissible (safe) region. In this work, we propose a systematic approach to guarantee the satisfaction of virtual workspace constraints in 6D for arbitrary manipulator dynamics based on an extended invariance control concept. Invariance control yields a computationally efficient method to render multiple virtual nonlinear workspace boundaries. In order to make the scheme suitable for pHRI we present an approach to reduce chattering by explicitly considering the discrete-time Euler solver output. Orientation constraints are unambiguously represented as unit quaternions. The theoretical results are successfully validated in simulation and experiments on a 7DoF anthropomorphic manipulator.
\end{abstract}

\section{INTRODUCTION}

Many application domains for robots require the ability to interact closely or even physically with humans. Among them are mobility assistance for elderly people and cooperative object manipulation in domestic and industrial settings, see e.g. [1]. One crucial characteristic of pHRI is the physical coupling of human and robot - direct or through an object. It is well-known that the interplay of compliance, safety, performance, and dependability under real-time conditions is key to success in pHRI [2]. We aim to address these fundamental prerequisites of pHRI within a single control framework combining active compliance control [3] with the enforcement of virtual workspace constraints restricting the manipulator motion to a safe region.

The topic of virtual workspace constraints is related to haptic virtual-wall rendering as addressed in the virtual reality literature. In order to render a haptic virtual wall in a virtual environment, the admittance characteristics of the admittance-controlled haptic interface switches from freespace (zero stiffness) to a very stiff characteristics at the virtual wall boundary [4]. Also related is the topic of forbiddenregion virtual fixtures from telerobotics, which keep the manipulator out of a certain workspace region [5]. Depending on the impedance/admittance-type of the forbidden-region virtual fixture either a high force proportional to the penetration depth is generated similar to a virtual wall or any motion command driving the teleoperator into a forbidden region is rejected [6]. However, the approaches mentioned above do not consider the dynamics of the robotic system approaching the constraint and therefore cannot ensure to avoid a penetration into the constraints.
In this paper we propose invariance control as a generic and systematic scheme to enforce robot workspace constraints in 6D. Invariance control [7], overcomes the above mentioned limitations of existing approaches by taking the system's (potentially nonlinear) dynamics and constraints on the actuating variables explicitly into account [8]. Advantageously, it yields a computationally efficient method to render multiple virtual nonlinear workspace boundaries; within the admissible region the nominal control scheme is active. To the best of our knowledge, invariance control has not yet been considered to render workspace constraints in autonomous robotics. Cooperative tasks may include constraints on the position as well as on the orientation in 3-dimensional space. This includes problems of collision avoidance and self-collision avoidance as addressed by [9], and [10]. We investigate invariance control on poses in $\mathbb{R}^{3} \times \mathbb{R P}^{3}$, which is not addressed in the literature.

Nevertheless, while ensuring the positive invariance property of the admissible region, the approach suffers from chattering effects at the boundaries of the admissible region. The chattering induces stable oscillations close to the boundary, which is particularly undesirable in pHRI as the vibration effect is directly exerted on the human interaction partner.

The contribution of this paper is an invariance control concept to enforce robot workspace constraints in 6D with a particular focus on its application in physical humanrobot interaction. This is achieved by presenting orientation constraints unambiguously as unit quaternions. An active compliance control is embedded as a nominal control within the admissible (safe) region of the workspace. The chattering reduction method proposed in this work preserves the properties of invariance control while enhancing the quality of interaction close to the workspace boundaries for physical human-robot interaction. Chattering reduction is achieved by explicit consideration of the discrete-time Euler solver output. Both contributions are successfully validated in simulation and on an anthropomorphic manipulator with 7 degrees of freedom (DoF).

The remainder of this paper is organized as follows: Section II gives the necessary background on invariance control. The extension to $6 \mathrm{D}$ pose constraints is presented in Section III and the novel chattering reduction method is introduced in Section IV. Simulations are provided in Section V, experiments in Section VI. 
Notation: In the following, bold characters are used for vectors and matrices. Low order time derivatives are denoted by dots $\left(\ddot{y}:=\frac{\mathrm{d}^{2} y}{\mathrm{~d} t^{2}}\right)$. Higher order derivatives are denoted $y^{(i)}:=\frac{\mathrm{d}^{i} y}{\mathrm{~d} t^{2}}$. The directional derivative of the scalar function $h(\boldsymbol{x})$ in the direction of $\boldsymbol{f}$ is the Lie-Derivative

$$
\mathcal{L}_{\boldsymbol{f}} h(\boldsymbol{x})=\frac{\partial h}{\partial \boldsymbol{x}} \boldsymbol{f} .
$$

Lie-Derivatives $\left(\mathcal{L}_{f}^{i}\right)$ of higher order $i$ are defined recursively. The Euclidean norm (2-norm) of vector $\boldsymbol{u}$ is denoted $\|\boldsymbol{u}\|_{2}$. The Moore-Penrose generalized inverse of matrix $\boldsymbol{A}$ is denoted by $\boldsymbol{A}^{+}=\boldsymbol{A}^{T}\left(\boldsymbol{A} \boldsymbol{A}^{T}\right)^{-1}$ and $\boldsymbol{A} \boldsymbol{A}^{+}=I$.

\section{INVARIANCE CONTROL}

Due to space limitations, only a brief introduction to the concept of invariance control is given in this section. We kindly refer the reader to [11], [12] for a more complete overview on the fundamentals of invariance control.

\section{A. Basic Principles}

The invariance controller supervises a nominal controller and corrects the control output if and only if necessary to ensure that the system state remains within a pre-defined positive invariant set, independent of the future reference applied to the nominal control. For the sake of simplicity, we introduce the concept for the single-input singleoutput (SISO) case first before the multi-input multi-output (MIMO) case is treated in Section II-B. Any nonlinear, control affine system

$$
\dot{\boldsymbol{x}}=\boldsymbol{f}(\boldsymbol{x})+\boldsymbol{g}(\boldsymbol{x}) u \quad \text { with } \boldsymbol{x}(t=0)=\boldsymbol{x}_{\mathbf{0}},
$$

with a state vector $\boldsymbol{x} \in \mathbb{R}^{n}$, a scalar control input $u \in \mathbb{R}$ and sufficiently smooth functions $f: \mathbb{R}^{n} \rightarrow \mathbb{R}^{n}$ and $\boldsymbol{g}: \mathbb{R}^{n} \rightarrow \mathbb{R}^{n}$ can be controlled using invariance control. Invariance control makes the system (1) controlled invariant with respect to a positively invariant set [13], defined by the function $\Phi(\boldsymbol{x})$

$$
\begin{aligned}
\mathcal{G} & =\{\boldsymbol{x} \mid \Phi(\boldsymbol{x}) \leq 0\} \\
\partial \mathcal{G} & =\{\boldsymbol{x} \mid \Phi(\boldsymbol{x})=0\} .
\end{aligned}
$$

This invariance function $\Phi(\boldsymbol{x})$ needs to be decreasing on the boundary $\partial \mathcal{G}$ for the region $\mathcal{G}$ to be invariant. The proof for this condition can be found in [13].

The basic structure of a system with invariance control is shown in Fig. 1. The nominal control is designed to ensure the achievement of the over-all control goal without considering the constraints. At times, when no constraint is violated, this nominal control determines the dynamic behavior and performance of the system. A switching policy enables the corrective controller to prevent a violation of the constraints.

In order to derive the invariance function, first the admissible set for the system state is defined by means of an output function for each constraint

$$
y_{i}=h_{i}(\boldsymbol{x}) \leq 0 \quad \text { for } 1 \leq i \leq m,
$$

resulting in the admissible set

$$
\mathcal{H}=\{\boldsymbol{x} \mid h(\boldsymbol{x}) \leq 0\} .
$$

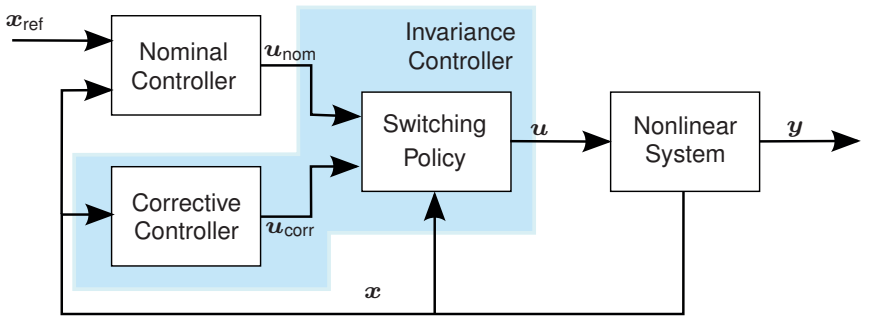

Fig. 1. Basic structure of an invariance controlled system

The invariance function also depends on the relative degree $r$ of the system (1), which is determined by input-outputlinearizing the system using the Lie-derivative

$$
\begin{array}{ll} 
& \stackrel{(r)}{y}=\mathcal{L}_{\boldsymbol{f}}^{r} h(\boldsymbol{x})+\mathcal{L}_{\boldsymbol{g}} \mathcal{L}_{\boldsymbol{f}}^{r-1} h(\boldsymbol{x}) u \\
\text { with } & \mathcal{L}_{\boldsymbol{g}} \mathcal{L}_{\boldsymbol{f}}^{i} h(\boldsymbol{x})=0 \text { for } 0<i<r-1 \\
\text { and } & \mathcal{L}_{\boldsymbol{g}} \mathcal{L}_{\boldsymbol{f}}^{r-1} h(\boldsymbol{x}) \neq 0 .
\end{array}
$$

The invariance function is then given by

$$
\begin{array}{ll}
r=1: & \Phi(\boldsymbol{x})=y \\
r=2: & \Phi(\boldsymbol{x})=\left\{\begin{aligned}
y & \text { if } \dot{y} \leq 0 \\
-\frac{1}{2 \gamma} \dot{y}^{2}+y & \text { if } \dot{y}>0 .
\end{aligned}\right.
\end{array}
$$

As long as this invariance function has a value smaller than zero, the system is not in danger of violating a constraint and the nominal control can be applied.

\section{B. Multi-Input Multi-Output Systems}

Since there is more than one control input in Multi-Input Multi-Output (MIMO) systems, compliance with multiple constraints is possible [8]. For each constraint, an output function needs to be defined and these functions can be merged into a vector

$$
\boldsymbol{y}=\boldsymbol{h}(\boldsymbol{x}) \Leftrightarrow\left\{\begin{array}{l}
y_{1}=h_{1}(\boldsymbol{x}) \\
\vdots \\
y_{l}=h_{l}(\boldsymbol{x}) .
\end{array}\right.
$$

The relative degree (5) has to be determined for each element of this vector, which then defines the so-called pseudo input

$$
z_{i}=\stackrel{\left(r_{i}\right)}{y_{i}}=\boldsymbol{a}_{i}{ }^{T}(\boldsymbol{x}) \boldsymbol{u}+b_{i}(\boldsymbol{x})
$$

$$
\begin{aligned}
\text { with } \boldsymbol{a}_{i}^{T}(\boldsymbol{x}) & =\left[\mathcal{L}_{\boldsymbol{g}_{\mathbf{1}}} \mathcal{L}_{\boldsymbol{f}}^{r_{i}-1} h_{i}(\boldsymbol{x}) \cdots \mathcal{L}_{\boldsymbol{g}_{\boldsymbol{m}}} \mathcal{L}_{\boldsymbol{f}}^{r_{i}-1} h_{i}(\boldsymbol{x})\right] \\
\text { and } b_{i}(\boldsymbol{x}) & =\mathcal{L}_{\boldsymbol{f}}^{r} h_{i}(\boldsymbol{x}),
\end{aligned}
$$

which can be compactly rewritten by combining the pseudo inputs to a vector

$$
\begin{gathered}
\boldsymbol{z}=\boldsymbol{b}(\boldsymbol{x})+\boldsymbol{A}(\boldsymbol{x}) \boldsymbol{u} \\
\text { with } \boldsymbol{A}(\boldsymbol{x})=\left[\begin{array}{c}
\boldsymbol{a}_{1}^{T} \\
\vdots \\
\boldsymbol{a}_{l}^{T}
\end{array}\right], \boldsymbol{b}(\boldsymbol{x})=\left[\begin{array}{c}
\mathcal{L}_{\boldsymbol{f}}^{r_{1}} h_{1}(\boldsymbol{x}) \\
\vdots \\
\mathcal{L}_{\boldsymbol{f}}^{r_{l}} h_{l}(\boldsymbol{x}),
\end{array}\right]
\end{gathered}
$$

and the parameter $\boldsymbol{a}_{i}$ defined as in (8). Since the constraints are used to bound the workspace in our setting, not all constraints are about to be violated at all times. Therefore 
the notion of an active constraint is introduced describing those outputs, in which the system is about to leave the admissible set. The set $\mathcal{K}(\boldsymbol{x})$ contains the indices of the active constraints. It is assumed, that each pseudo input is bounded, which must hold true for active constraints and can be fulfilled by use of an input-output-linearizing control law.

$$
z_{i} \leq \gamma_{i} \quad \forall i \in \mathcal{K}(\boldsymbol{x})
$$

Using this constraint on the pseudo input, (8) can be rewritten into the element-wise inequality

$$
\boldsymbol{A}_{\mathcal{K}}(\boldsymbol{x}) \boldsymbol{u} \leq \boldsymbol{b}_{\mathcal{K}}(\boldsymbol{x})
$$

where $\boldsymbol{A}_{\mathcal{K}}(\boldsymbol{x})$ is derived from the matrix $\boldsymbol{A}(\boldsymbol{x})$ by only considering those lines that correspond to active constraints and

$$
\boldsymbol{b}_{\mathcal{K}}(\boldsymbol{x})=\left[\begin{array}{c}
\gamma_{i_{1}}-b_{i_{1}}(\boldsymbol{x}) \\
\vdots \\
\gamma_{i_{k}}-b_{i_{k}}(\boldsymbol{x})
\end{array}\right] \text {. }
$$

Similarly, for the vector $\boldsymbol{b}_{\mathcal{K}}(\boldsymbol{x})$ only those elements from $\boldsymbol{b}(\boldsymbol{x})$ corresponding to active constraints are used. More details on invariance control in MIMO systems are found in [8].

\section{Corrective Control}

It is desirable that the control $\boldsymbol{u}$ deviates least possible from the nominal control input $\boldsymbol{u}_{\text {nom }}$ but keeps the system controlled invariant. Therefore, we pose the search for the control as minimization problem

$$
\min \left\|\boldsymbol{u}-\boldsymbol{u}_{\text {nom }}\right\|_{2}^{2}
$$

which can be solved analytically if the number of active constraints is limited to the number of degrees of freedom, i.e. $\operatorname{dim} \boldsymbol{u} \geq \operatorname{dim} \boldsymbol{y}$. Note that in case of workspace constraints this is a reasonable assumption. This results in a side condition to the optimization problem [8]

$$
\begin{gathered}
\min \left\|\boldsymbol{u}-\boldsymbol{u}_{\text {nom }}\right\|_{2}^{2} \\
\text { s. t. } \boldsymbol{A}(\boldsymbol{x})\left(\boldsymbol{u}-\boldsymbol{u}_{\mathrm{nom}}\right)-\left(\boldsymbol{z}-\boldsymbol{b}(\boldsymbol{x})-\boldsymbol{A}(\boldsymbol{x}) \boldsymbol{u}_{\mathrm{nom}}\right)=0 .
\end{gathered}
$$

The constraint in the optimization problem ensures that nominal control is used in directions where no constraint is active. The value of the pseudo control input $z$ depends on the active constraints and is determined by

$$
z_{i}= \begin{cases}z_{n o m, i} & \text { if }\left\{\begin{array}{l}
\phi_{i}<0 \\
\vee\left(\dot{h}_{i}<0 \wedge z_{n o m, i} \leq 0\right) \\
\vee z_{n o m, i} \leq \gamma_{i}
\end{array}\right. \\
0 \quad & \text { if } \dot{h}_{i}<0 \wedge z_{n o m, i}>0 \\
z_{\text {corr }, i}=\gamma_{i} & \text { otherwise }\end{cases}
$$

with $z_{\text {nom }}$ determined as in (9). The control output is then determined by

$$
\boldsymbol{u}=\boldsymbol{A}^{+}(\boldsymbol{x})\left(\boldsymbol{z}-\boldsymbol{b}(\boldsymbol{x})-\boldsymbol{A}(\boldsymbol{x}) \boldsymbol{u}_{\mathrm{nom}}\right)+\boldsymbol{u}_{\mathrm{nom}} .
$$

As $z_{i}$ switches at the boundary, chattering may occur and cause undesirable oscillations. We propose an approach to reduce chattering in Section IV.

\section{Manipulator InVARIANCE CONTROL WiTh 6D POSE CONSTRAINTS}

In this Section we derive a invariance control scheme to enforce robotic manipulator workspace constraints in 6D.

\section{A. Nominal Control}

The first step in setting up an invariance control law is the design of the nominal control, that ensures the achievement of the overall control goal. In order to render an active compliance as desirable for pHRI we consider an admittancebased control as the nominal one rendering the end-effector dynamics in Cartesian space to

$$
\ddot{\boldsymbol{p}}_{d}=\boldsymbol{M}_{p}^{-1}\left(\boldsymbol{f}-\boldsymbol{D}_{p} \dot{\boldsymbol{p}}_{d}-\boldsymbol{K}_{p} \boldsymbol{p}_{d}\right)
$$

with the vector $\boldsymbol{p}_{d} \in \mathbb{R}^{3}$ describing the desired Cartesian position, the inertia matrix $M_{p}$, viscous friction $D_{p}$, and virtual spring $\boldsymbol{K}_{p}$.

The orientation is unambiguously presented as unit quaternion

$$
q=q_{0}+\mathrm{i} \cdot q_{1}+\mathrm{j} \cdot q_{2}+\mathrm{k} \cdot q_{3},
$$

with the elements $q_{i}$ of the unit quaternion describing the current orientation of the end effector. The dynamics of in the rotational degrees of freedom is given by

$$
\dot{\boldsymbol{\omega}}_{d}=\boldsymbol{M}_{o}^{-1}\left(\boldsymbol{\tau}-\boldsymbol{D}_{o} \boldsymbol{\omega}_{d}-2 \boldsymbol{Q} \boldsymbol{K}_{o}\left(\begin{array}{l}
q_{1} \\
q_{2} \\
q_{3}
\end{array}\right)\right),
$$

with the desired angular velocity $\boldsymbol{\omega}_{d}$, torques $\tau$ at the end effector, and

$$
\boldsymbol{Q}=\left(\begin{array}{rrr}
q_{0} & -q_{3} & q_{2} \\
q_{3} & q_{0} & -q_{1} \\
-q_{2} & q_{1} & q_{0}
\end{array}\right)
$$

as introduced in [14]. Inertia $M_{o}$, rotational damping $\boldsymbol{D}_{o}$, and a rotational spring $\boldsymbol{K}_{o}$ represent the rotational impedance parameters. The invariance controller with this nominal controller determines a desired trajectory for the robot that is compliant with the constraints even when force or torque is applied to the end effector. Note that other control schemes are also feasible for the nominal control.

\section{B. Constraints in $6 D$}

The invariance controller is supposed to constrain the workspace of the robot to a specific region. In the context of pHRI, typically pose constraints are required for safe interaction or for the interactive task. In the following, it is shown, how constraints and the corresponding output functions are defined for both the Cartesian coordinates and the orientation of the end effector. For simplicity of presentation we consider box constraints in Cartesian coordinates and simple angular constraints for the end effector. The extension to more complicated workspace boundaries is straightforward using (7).

Box constraints on the end effector's Cartesian position $\boldsymbol{p}$ in the robot's coordinate system are defined by the output function [8]

$$
\boldsymbol{h}_{p}=\left[\begin{array}{l}
\boldsymbol{p}_{\min }-\boldsymbol{p} \\
\boldsymbol{p}-\boldsymbol{p}_{\max }
\end{array}\right]
$$




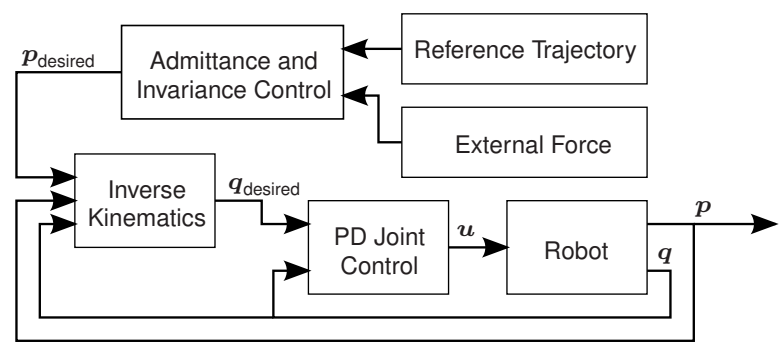

Fig. 2. Control structure for evaluation of the approach in simulation and experiments with an anthropomorphic manipulator

An angular constraint is defined to restrict the angle between an axis of the end effector coordinate system and the corresponding axis of the robot coordinate system to a predefined maximum value $\alpha_{\max }$. The corresponding output function

$$
h_{\alpha}=\alpha-\alpha_{\max }
$$

is used to calculate the matrix $\boldsymbol{A}(\boldsymbol{x})$ and the vector $\boldsymbol{b}(\boldsymbol{x})$ in (9). In order for the angular constraints to be unambiguous, the angle $\alpha$ is expressed using unit quaternions. In case of only one constraint, $\boldsymbol{A}(\boldsymbol{x})$ is a vector and $\boldsymbol{b}(\boldsymbol{x})$ a scalar value. For multiple constraints, as for the Cartesian constraints, the output functions have to be combined in a vector, so $\boldsymbol{A}(\boldsymbol{x})$ will be a matrix and $\boldsymbol{b}(\boldsymbol{x})$ becomes a vector. The resulting control law, which is determined using (13) and (14), ensures that the robot's end effector remains within the desired boundaries at all times, follows a given reference trajectory and is compliant to external forces. Its structure is depicted in Fig. 2.

\section{Chattering Reduction}

In real-time capable discrete-time implementations, invariance control suffers from chattering [8]. Chattering occurs during the motion along a boundary. A constant value of the control parameter $\gamma$ results in repeated switching in the corrective control output. This is caused by alternating switching of the control input between corrective control and nominal control, depending on the invariance function value at the current time step. This leads to undesired vibrations adding discomfort and reduced interaction quality for the human. Therefore, a method that reduces chattering will is proposed that explicitly considers an Euler-type solver with sampling time $T_{A}$, which is used to evaluate control loop.

A movement along a boundary of the invariant set is determined by the fixed value of the control parameter $\gamma$ according to (13). There are two cases to be considered:

1) The boundary is not yet violated, but the invariance function has a value larger than zero.

2) The boundary is violated.

Figure 3(a) shows the behavior of an invariance controller with a fixed value for $\gamma$ in those two cases. The red line depicts those values of $h$ and $\dot{h}$ for which the invariance function is equal to zero. For all points that are to the right of this curve, the invariance function is larger than zero and corrective control is needed.

Corrective control decreases the change in the output function in the following time step. In case 1), this might

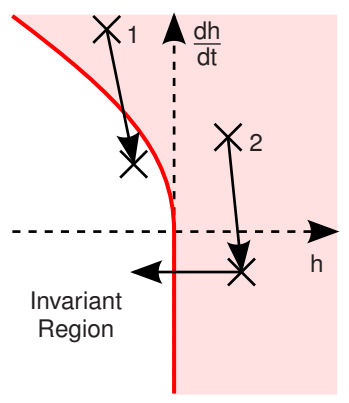

(a)

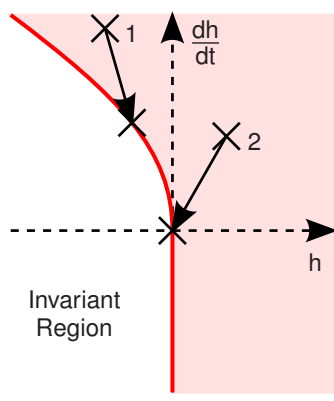

(b)
Fig. 3. Comparison of the behavior of an invariance controller with (a) a fixed parameter $\gamma$ (b) an adapted parameter $\gamma$

already be enough for the invariance function to equal a value larger than zero. In order to achieve sliding along the boundary, it would, however, be necessary to end up exactly on the red line, where the function is equal to zero. In case 2 ), a change in the rate of change of the output function alone is not enough to return into the invariant set. If the change is enough to result in a negative derivative of the output function, the value of the output function will return into the invariant set eventually, but again, it will not stop at the point, where the invariance function is equal to zero.

A more desirable behavior of the invariance controller is shown in Fig. 3(b). In case 1), the goal should be, to find a control output that will change the derivative of the output function in a way that the invariance function is reduced to zero in the following time step, whereas in in case 2) it has to be ensured, that the change in the output function is reduced to zero as well, when the output function itself is reduced. This motivates us to search for an appropriate modulation of $\gamma$ to achieve this behavior. In order to determine a convenient value for the corrective output in the first case,

$$
\Phi_{k+1}=\frac{1}{2 \gamma_{\text {adapted }}} \dot{h}_{k+1}^{2}+h_{k+1}=0
$$

has to be solved. This can be done using $h_{k+1}=h_{k}+T_{A} \dot{h}_{k}$ and $\dot{h}_{k+1}=\dot{h}_{k}+T_{A} \cdot \gamma_{\text {adapted. }}$. After solving the quadratic equation for $\gamma$, this leads to the following two values:

$$
\gamma_{\text {adapted }, \pm}=\frac{h \pm \sqrt{h^{2}-4 \cdot T_{A}^{2} \cdot \dot{h}^{2}}}{T_{A}^{2}} .
$$

The less conservative smaller value of the two will be used for the invariance control. However, this solution cannot be used if

$$
h^{2}-4 \cdot T_{A}^{2} \cdot \dot{h}^{2}<0
$$

holds true, which might happen close to a boundary, since then the square root in (19) does not have a real value. In this case or if a boundary is already violated, another approach has to be taken. The main goal then should be, to return to the boundary and to reduce the rate of change of the corresponding output function to zero. In order to reduce the required acceleration, this adjustment will be made not in one time step $T_{A}$ but in a time interval $n T_{A}$. The value 
for $\gamma$ that reduces the rate of change to zero in $\mathrm{n}$ time steps is determined by

$$
\gamma_{\text {adapted,vel }}=-\frac{\dot{h}}{n T_{A}} .
$$

For the return to the boundary, the required control input is only applied in the first time step but the boundary will be reached in $\mathrm{n}$ time steps. So for the (n-1) following time steps, the velocity is assumed to be constant. The required value for $\gamma$ can then be determined by solving

$$
\begin{aligned}
p_{k+n} & =p_{k+1}+(n-1) T_{A} \dot{p}_{k+1} \\
& =p_{k}+T_{A} \dot{p}_{k}+(n-1) T_{A}\left(\dot{p}_{k}+T_{A} \gamma_{\text {adapted,pos }}\right),
\end{aligned}
$$

where $p$ is the bounded coordinate. Since the goal is to reach the boundary, $p_{k+n}=p_{\max }$ has to hold and $h=$ $p_{k}-p_{\max }, \dot{h}=\dot{p}$ can be substituted, which results in

$$
\gamma_{\text {adapted,pos }}=-\frac{h+n T_{A} \dot{h}}{(n-1) T_{A}^{2}} .
$$

These two values for $\gamma$ from (20) and (21) have to be combined to achieve both goals. Therefore a convex combination of the values of $\gamma$ is used:

$$
\gamma_{\text {adapted }}=g \cdot \gamma_{\text {adapted,vel }}+(1-g) \cdot \gamma_{\text {adapted,pos }}
$$

The pseudo control output from (13) can be replaced using (19) and (22).

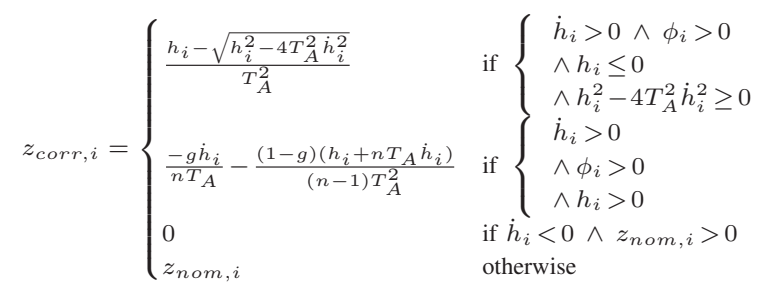$$
\text { if } z_{c o r r, i}<\gamma_{\min } \text { then } z_{c o r r, i}=\gamma_{\min } \text {. }
$$

Still, condition (24) must be satisfied for bounded control inputs $\boldsymbol{u}$ as real motors are not able to create an arbitrary acceleration. With the pseudo input from (23), chattering in the movement along a boundary can be reduced significantly.

\section{Simulation}

The validity and performance of the proposed invariance control concept with chattering reduction is evaluated in simulations using MATLAB/Simulink of a robotic manipulator with the control structure given in Fig. 2. The model consists of the part, where invariance control influences the admittance controller in a way such that the resulting desired position and orientation of the end effector is constraint admissible and a model of a closed-loop controlled robot manipulator. The admittance and invariance control block combines the effects of external forces and torques with the reference trajectory using the admittance controller in (15) and (16) and checks the result for compliance with the boundaries by using (23) or (13) and if necessary by adjusting the input of the admittance controller with (14). The closed loop ensures that an admissible trajectory is followed. For simulation purposes, the robot is modelled as PD-controlled, damped double integrator system. In the
TABLE I

SIMULATION PARAMETERS

\begin{tabular}{lll}
\hline Sampling time & $T_{A}$ & $0.001 \mathrm{~s}$ \\
Cartesian impedance & $\boldsymbol{K}_{p}$ & $600 \mathrm{~N} / \mathrm{s} \cdot \boldsymbol{I}_{3}$ \\
Rotational impedance & $\boldsymbol{K}_{o}$ & $20 \mathrm{~N} / \mathrm{rad} \cdot \boldsymbol{I}_{3}$ \\
Cartesian damping & $\boldsymbol{D}_{p}$ & $80 \mathrm{Ns} / \mathrm{m} \cdot \boldsymbol{I}_{3}$ \\
Rotational damping & $\boldsymbol{D}_{o}$ & $7 \mathrm{Ns} / \mathrm{rad} \cdot \boldsymbol{I}_{3}$ \\
Lower position constraint & $x_{\min }$ & $0.57 \mathrm{~m}$ \\
Upper position constraint & $x_{\max }$ & $0.7 \mathrm{~m}$ \\
Upper orientation constraint & $\alpha_{\max }$ & $9^{\circ}$ \\
Cartesian control parameter & $\gamma_{\text {cart }}$ & -18 \\
Rotational control parameter & $\gamma_{\text {rot }}$ & -12 \\
\hline
\end{tabular}

simulation, only constraints on the x-position are used and the controlled angle is the deviation of the y-axis in the robot coordinate system from the end effector's y-axis. Therefore, the angle $\alpha$ in (18) is determined by

$$
\tan (\alpha)=\frac{\sqrt{q_{1, x}^{2}+q_{3, x}^{2}}}{q_{2, x}}
$$

with $q_{i, x}$ as the elements of the quaternion rotation

$$
q_{x}=q \cdot \mathrm{j} \cdot q^{-1} .
$$

The simulation parameters for the following results are given in Table I. Figure 4 as well as Fig. 5 show the results of the simulation with the conventional invariance control. It is observed that there is an adherence to the Cartesian workspace boundary and that the end effector follows the admissible trajectory reasonably well. However, at the Cartesian boundary oscillations occur, see Fig. 4. Also, the angular constraint is violated due to a strong oscillation, see Fig. 5. Successful chattering reduction with the novel

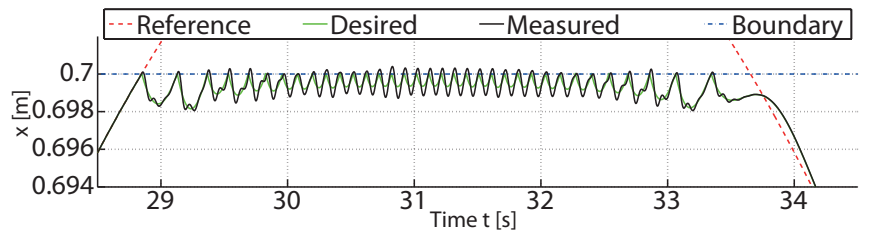

Fig. 4. Chattering at the Cartesian $x$-boundary with conventional invariance control without chattering reduction

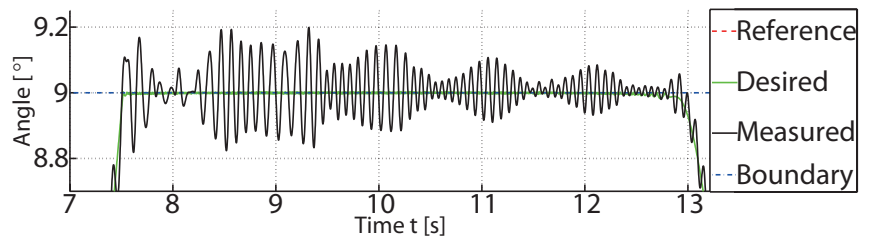

Fig. 5. Chattering at angular constraint with conventional invariance control

method is presented in Fig. 6 and Fig. 7 in contrast to the same scenario and a conventional invariance control as shown in Fig. 4 and Fig. 5. The chattering reducing approach from (23) and (24) is used in simulation. The figures show a significant reduction of the oscillations. Apart from an overshoot and a resulting transient effect, the admissible trajectory runs precisely along the boundary. In this case, the adherence of the end effector's position and orientation 


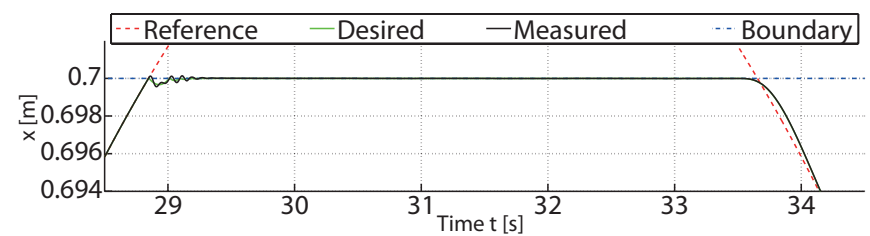

Fig. 6. Significantly reduced chattering at the Cartesian $x$-boundary with novel invariance control with chattering reduction

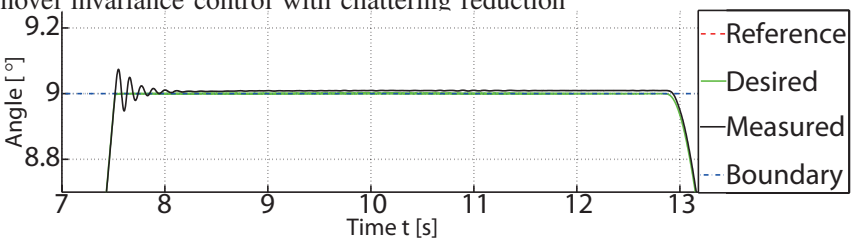

Fig. 7. Significantly reduced chattering at angular constraint with novel invariance control with chattering reduction

to the constraints depends only on its ability to follow the desired trajectory. The difference between a conventional invariance control and the novel control approach with an adaptive parameter $\gamma$ can be observed by comparing Fig. 8 and Fig. 9. The figures show the pseudo control input that is created at the approach of the boundary shown in Fig. 5 and 7. Figure 8 depicts the input created by the conventional invariance controller. There, the pseudo input only takes two values, either $-12 \frac{\mathrm{m}}{\mathrm{s}^{2}}$ or $1 \frac{\mathrm{m}}{\mathrm{s}^{2}}$, while the adapted invariance controller also creates values in between as shown in Fig. 9.

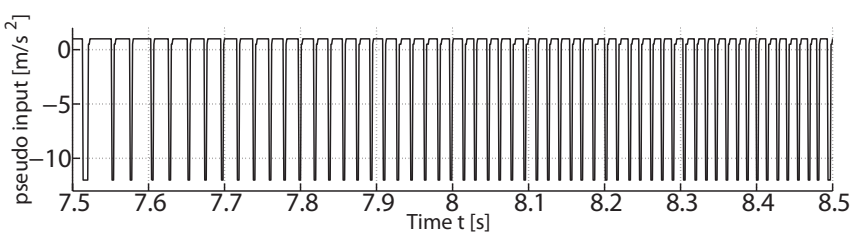

Fig. 8. Pseudo control input created by conventional invariance controller

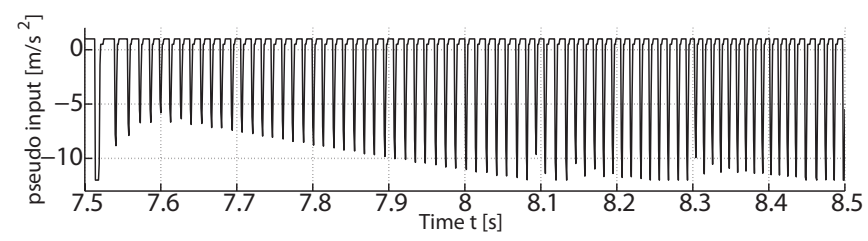

Fig. 9. Pseudo control input with adaption of $\gamma$

\section{EXPERIMENTAL EVALUATION}

As shown in Section V, the effect of chattering is successfully reduced with our proposed approach in simulation and the effectiveness on an actual electromechanical system in interaction with a human partner is experimentally evaluated.

\section{A. Experimental Setup}

The experiment is carried out on an anthropomorphic manipulator with seven degrees of freedom [15], [16] depicted in Fig. 10. The control algorithm is running on Linux/PreemptRT using Matlab/Simulink's Real-Time Workshop at a sampling rate of $1 \mathrm{kHz}$ using a discrete-time Euler

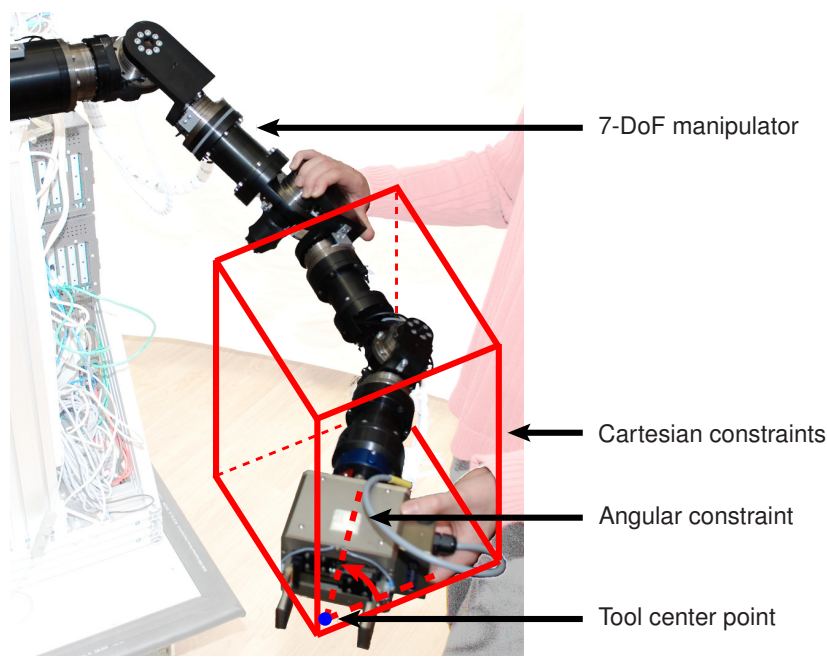

Fig. 10. Anthropomorphic manipulator in interaction pushed to two positional and one orientational virtual constraint

solver. The applied human force $\boldsymbol{F}$ is measured at the wrist using a JR3 sensor. The end effector position is determined through closed-loop differential inverse kinematics from joint position encoders with 1024 ticks per motor revolution. Harmonic Drive gears with transmission ratios of $1: 100$ in shoulder and elbow joints (J1 to J4) and $1: 160$ in the wrist (J5 to J7) ensure backlash-free interaction. In addition to the parameters and constraints given in Table I, two additional cartesian box constraints are introduced in the experiment: $y \in[0.13 \mathrm{~m}, 0.4 \mathrm{~m}]$, and $z \in[-0.5 \mathrm{~m},-0.35 \mathrm{~m}]$.

\section{B. Experimental Results}

For the experiment, the constraints are chosen to be box constraints in the Cartesian coordinates and maximum angular deviation of the $y$-axis of the end effector from the robot's $y$-axis. During the experiment, forces and torques of random direction and magnitude are applied to the end effector. The resulting trajectory in the Cartesian coordinates is shown in Fig. 11. It can be observed that the green desired trajectory is always compliant with the boundaries, whereas the position of the end effector, depicted in black, is not. This happens when the applied force, as shown in Fig. 12 reaches a value which the actuators of the robot arm cannot compensate, since they are not able to create arbitrary counterforces. As soon as the force is reduced, however, the position of the end effector is adjusted immediately. It can also be observed, that when no force is applied, the end effector exactly follows the desired trajectory. The orientation of the end effector shows a similar behavior. Fig. 13 depicts the deviation of the end effector's y-axis from the robot's y-axis, when the torque from Fig. 14 is applied. In this case, the trajectory is compliant with the boundary, since the applied torque is small enough for the actuators to counteract. When no torque is applied, the y-axis aligns itself with the system's y-axis and the angle is reduced to zero. 

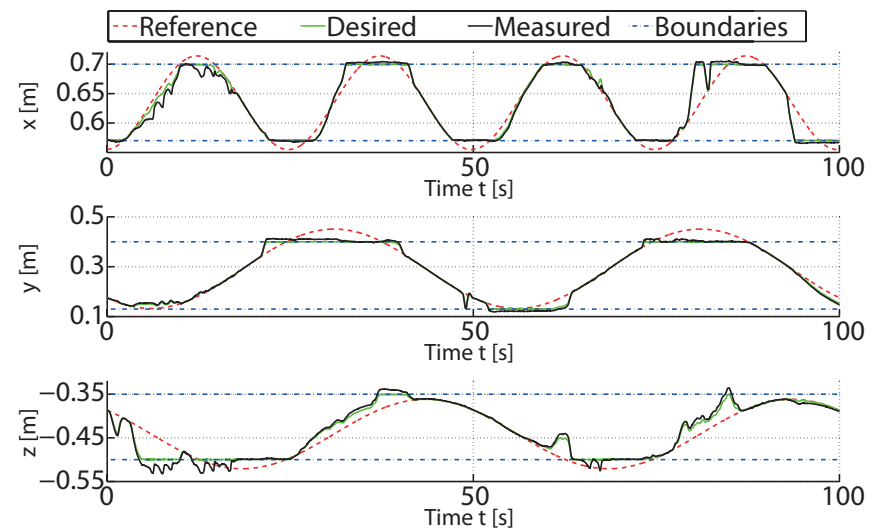

Fig. 11. End effector trajectory in $x, y$ and $z$ coordinates, bounded in each coordinate using invariance control
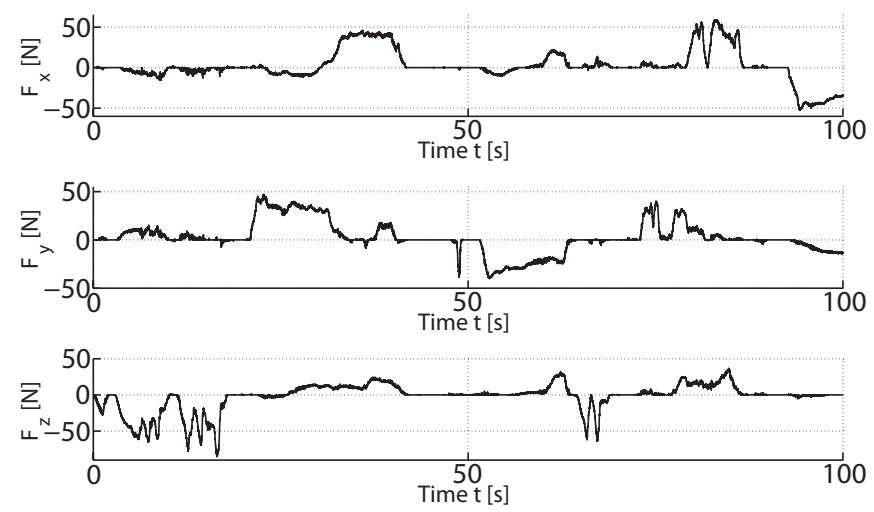

Fig. 12. Human applied force causing a deviation from the reference trajectory in the Cartesian coordinates

\section{CONCLUSION}

In this work, we propose a novel control approach to constrain the robot workspace in 6D with focus on application in physical human-robot interaction based on invariance control. The manipulator dynamics is explicitly considered to avoid penetration of the boundaries at high speed. Arbitrary smooth workspace constraints can be considered. We also propose a chattering reduction approach to reduce disturbing oscillations along the workspace boundaries. In experiments and simulations the scheme is shown to be able to satisfy $6 \mathrm{D}$ workspace constraints while effectively preserving the desired compliant behavior of the robot manipulator within the admissible region. In conclusion, invariance control is a very promising, compuationally efficient tool to execute (cooperative) manipulation tasks involving compliant robot behavior while obeying safety- or task-relevant constraints. A remaining challenge is the inclusion of constraints on jointlevel. Therefore, our future work targets the extension to mixed joint- and work-space constraints in one framework.

\section{ACKNOWLEDGEMENTS}

This work is supported in part within the DFG excellence initiative research cluster Cognition for Technical Systems CoTeSys (www. cotesys.org).

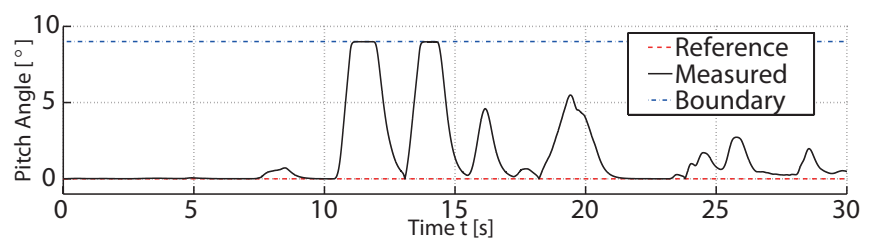

Fig. 13. Change of the angle between the robot's and the end effector's $\mathrm{y}$-axis, bounded using invariance control

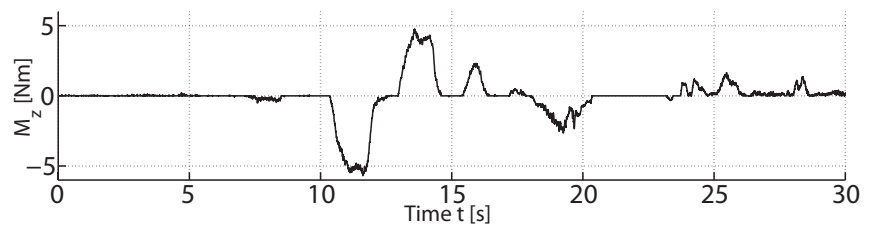

Fig. 14. Applied torque in z-direction causing a change in the end effector's orientation

\section{REFERENCES}

[1] J. Medina, M. Lawitzky, A. Mörtl, D. Lee, and S. Hirche, "An Experience-Driven Robotic Assistant Acquiring Human Knowledge to Improve Haptic Cooperation," in Proc. IEEE/RSJ IROS, 2011, pp. $2416-2422$.

[2] A. DeSantis, B. Siciliano, A. DeLuca, and A. Bicchi, "An atlas of physical humanrobot interaction," J. Mech. Mach. Theory, vol. 43, no. 3, pp. 253-270, 2008.

[3] N. Hogan, "Impedance Control: An approach to manipulation," J. Dyn. Sys., Meas., Control, vol. 107, pp. 1-24, 1985.

[4] B. Gillespie and M. Cutkosky, "Stable User-Sepcific Haptic Rendering of the Virtual Wall," in Proc. ASME International Mechanical Engineering Congress \& Exposition, vol. 58, 1996, pp. 397-406.

[5] J. J. Abbott, P. Marayong, and A. M. Okamura, "Haptic Virtual Fixtures for Robot-Assisted Manipulation," in Robotics Research, R. B. S. Thrun and H. D. Whyte, Eds. Springer STAR series, 2007, pp. 49-64.

[6] S. Park, R. D. Howe, and D. F. Torchiana, "Virtual fixtures for robotic cardiac surgery," in Proc. MICCAI, 2001, pp. 1419-1420.

[7] J. Mareczek, M. Buss, and M. Spong, "Invariance Control of Normal Forms with Input Driven Internal Dynamics," in Proc. ACC, 2001, pp. 3648-3653.

[8] M. Scheint, J. Wolff, and M. Buss, "Invariance Control in Robotic Applications: Trajectory Supervision and Haptic Rendering," in Proc. ACC, 2008, pp. 1436-1442.

[9] F. Seto, K. Kosuge, and Y. Hirata, "Self-collision Avoidance Motion Control for Human Robot Cooperation System using RoBE," in Proc. IEEE/RSJ IROS, 2005, pp. 3143-3148.

[10] O. Schrempf, U. Hanebeck, A. Schmid, and H. Wörn, "A Novel Approach to Proactive Human-Robot Cooperation," in Proc. IEEE RoMan, 2005, pp. 555-560.

[11] J. Mareczek, M. Buss, and G. Schmidt, "Sufficient Conditions for Invariance Control of a Class of Nonlinear Systems," in Proc. IEEE CDC, December 2000, pp. 1436-1442.

[12] J. Wolff and M. Buss, "Invariance Control Design For Constrained Nonlinear Systems," in Proc. NOLCOS, 2004, pp. 711-716.

[13] _ - "Invariance Control Design For Nonlinear Control Affine Systems Under Hard State Constraints," in Proc. IFAC, 2005.

[14] F. Caccavale, C. Natale, B. Siciliano, and L. Villani, "Six-dof impedance control based on angle/axis representations," IEEE Trans. Robot. Automat., vol. 15, no. 2, pp. 289-300, April 1999.

[15] B. Stanczyk and M. Buss, "Development of a Telerobotic System for Exploration of Hazardous Environments," in Proc. IEEE/RSJ IROS, 2004, pp. 2532-2537.

[16] B. Stanczyk, "Development and Control of an Anthropomorphic Telerobotic System," Ph.D. dissertation, Technische Universität München, 2006. 